\title{
Correction to: Long Noncoding RNAs as Players in Breast Tumorigenesis
}

\author{
Cyrinne Achour and Francesca Aguilo
}

\section{Correction to:}

Chapter 15 in: S. Jurga, J. Barciszewski (eds.), The Chemical Biology of Long Noncoding RNAs, RNA Technologies 11, https://doi.org/10.1007/978-3-030-44743-4_15

The original version of chapter 15 was inadvertently published with an error in the chapter title. The correct chapter title is mentioned below and the chapter has now been corrected.

Chapter title: 'Long Noncoding RNAs as Players in Breast Tumorigenesis' 